\title{
Does assessment of signs and symptoms add to the predictive value of an algorithm to rule out pregnancy?
}

\author{
John Stanback, Nuru Nakintu, Zahida Qureshi, Marlina Nasution
}

\begin{abstract}
Background A World Health Organization-endorsed algorithm, widely published in international guidance documents and distributed in the form of a 'pregnancy checklist', has become a popular tool for ruling out pregnancy among family planning clients in developing countries. The algorithm consists of six criteria excluding pregnancy, all conditional upon a seventh 'master criterion' relating to signs or symptoms of pregnancy. Few data exist on the specificity to pregnancy among family planning clients of long-accepted signs and symptoms of pregnancy. The aim of the present study was to assess whether reported signs and symptoms of pregnancy add to the predictive value of an algorithm to rule out pregnancy.
\end{abstract}

Methods Data from a previous observational study were used to assess the performance of the algorithm with and without the 'signs and symptoms' criterion. The study group comprised 1852 new, non-menstruating family planning clients from seven clinics in Kenya.

Results Signs and symptoms of pregnancy were rare $(1.5 \%)$ as was pregnancy $(1 \%)$. Signs and symptoms were more common (18.2\%) among the 22 clients who tested positive for pregnancy than among the 1830 clients $(1.3 \%)$ who tested negative, but did not add significantly to the predictive value of the algorithm. Most women with signs or symptoms were not pregnant and would have been unnecessarily denied a contraceptive method using the current criteria.

Conclusions The 'signs and symptoms' criterion did not substantially improve the ability of the algorithm to exclude pregnant clients, but several reasons (including use of the algorithm for intrauterine device clients) render it unlikely that the algorithm will be changed.

J Fam Plann Reprod Health Care 2006; 32(1): 27-29

(Accepted 23 September 2005)

\section{Key message points}

- Unnecessary denial of family planning to non-menstruating clients remains a serious problem in developing countries.

- A popular algorithm for ruling out pregnancy among family planning clients subordinates six criteria predictive of nonpregnancy to one 'master criterion' based on signs and symptoms predictive of pregnancy.

- In this analysis of reported signs and symptoms of pregnancy in a cohort of 1852 Kenyan family planning clients, the 'master criterion' did not add significantly to the predictive value of the algorithm.

Family Health International, Research Triangle Park, NC, USA John Stanback, PhD, Senior Associate

Marlina Nasution, PhD, Biostatistician

\section{Department of Obstetrics and Gynaecology, Makerere University, Kampala, Uganda}

Nuru Nakintu, MD, Lecturer

\section{Department of Obstetrics and Gynaecology, University of Nairobi, Nairobi, Kenya}

Zahida Qureshi, MB BS, M Med, Senior Lecturer

Correspondence to: Dr John Stanback, Family Health International, PO Box 13950, Research Triangle Park, NC 27709, USA. Tel: +1919544 7040. Fax: +19195447261.

E-mail: jstanback@fhi.org

\section{Background}

In many developing countries, family planning clients who present for services in the absence of menstrual bleeding are routinely denied effective contraception and are told to return to the clinic at the onset of menses. ${ }^{1}$ Providers worry that contraception can harm unrecognised pregnancies, but in doing so ignore the risks run by clients who leave the clinic without effective contraception. This widespread problem stems from the unavailability of pregnancy tests and providers' unwillingness or inability to effectively use a client history to rule out pregnancy.

Sending clients away without a contraceptive method is, in most cases, unnecessary. A simple algorithm for excluding pregnancy with a reasonable degree of certainty was developed in the early 1990s and now is included in many international guidance documents including consensus recommendations published by the World Health Organization. ${ }^{2}$ The algorithm consists of six simple conditions (Box 1), meeting any one of which is sufficient to be 'reasonably certain that the woman is not pregnant'. However, the algorithm is written so that these six criteria are contingent upon a seventh 'master' criterion: that the woman have 'no symptoms or signs' of pregnancy. [NB. In this context, 'signs' are evidence of possible pregnancy that are detected by the health care provider; 'symptoms' are evidence of possible pregnancy reported by the client.] This final criterion is problematic for two reasons.

First, no particular signs or symptoms are specified (although other guidance documents for family planning cite symptoms such as nausea and mood changes and signs such as uterine softness and breast tenderness). 3,4 Before the advent of cheap diagnostic tests, health care providers routinely relied on clinical signs and symptoms to help diagnose pregnancy. However, most of the symptoms described in the literature are of limited usefulness to the family planning provider because they are non-specific in early pregnancy and may only be useful indicators after $8-10$ weeks of amenorrhoea. ${ }^{3}$

Second, while the first six criteria predict who is not pregnant, they are effectively made subordinate to the final 'signs and symptoms' criterion, which is designed to predict who is pregnant. Given the many risks of unintended pregnancy, unnecessarily denying family planning services to large numbers of women based on one insensitive predictor of pregnancy that overrides six highly specific identifiers of 'non-pregnancy' might be counterproductive. To assess the utility of the 'signs and symptoms' criterion, we conducted a secondary analysis of previously collected data used to validate an existing

Box 1: Criteria used in the algorithm for excluding pregnancy

The provider can be reasonably certain that the woman is not pregnant

if she has no symptoms or signs of pregnancy and meets any of the

following criteria:

- has not had intercourse since last normal menses

- has been correctly and consistently using a reliable method of contraception

- is within the first 7 days after normal menses

- is within 4 weeks postpartum for non-lactating women

- is within the first 7 days post-abortion or miscarriage

- is fully or nearly fully breastfeeding, amenorrhoeic, and less then 6 months postpartum. 
ARTICLE

Table 1 Description and frequency of signs and symptoms of pregnancy

\begin{tabular}{lrl}
\hline Signs and symptoms & Women $(n)$ & $\begin{array}{l}\text { Women who were } \\
\text { pregnant }(n)\end{array}$ \\
\hline Uterine mass & 2 & 2 \\
Nausea, abdominal pain, spitting & 1 & 1 \\
Nausea/vomiting & 18 & 1 \\
Engorged breasts & 2 & 0 \\
Vomiting, engorged breasts & 1 & 0 \\
Missed periods for 2 months & 1 & 0 \\
'Giddiness' & 1 & 0 \\
'Laziness' & 1 & 0 \\
Total & 27 & 4 \\
\hline
\end{tabular}

pregnancy exclusion checklist. Below, we review the signs and symptoms reported in that study and assess whether they improve the predictive value of the current algorithm.

\section{Methods}

In 1997, two of the authors (J.S. and Z.Q.) collected information from 1852 new, non-menstruating family planning clients in seven clinics in Kenya. Of the sample $59 \%$ were postpartum and lactating, 37\% reported they were between menses, and $4 \%$ had undergone recent abortions. Before prescription of their chosen method of contraception [i.e. hormonal methods or the intrauterine device (IUD)], each client was administered a checklist that included six questions based on the recommended criteria for ruling out pregnancy, as well as a space for providers to note any 'signs or symptoms of pregnancy'. Each client also provided urine for a pregnancy test. Details of the study are available elsewhere, 5 but the principal finding was that the low (1\%) pregnancy rate among family planning clients was responsible for the checklist's nearuniversal effectiveness at successfully ruling out pregnancy among non-pregnant clients.

For the present secondary analysis we reviewed and classified all the signs and symptoms of pregnancy noted in the original study and assessed their association with pregnancy. Then, to assess the potential impact of removing the 'signs and symptoms' criterion, we conducted a sensitivity analysis, comparing the validity of the checklist (sensitivity, specificity, positive and negative predictive value) with and without a master 'signs and symptoms' criterion.

\section{Results}

Overall, signs and symptoms of pregnancy were noted for only $27(1.5 \%)$ of the 1852 new, non-menstruating clients in the dataset (Table 1). [NB. Twenty of the 27 women met one or more of the other six criteria; seven did not.] Signs and symptoms were more common (18.2\%) among the 22 clients who tested positive for pregnancy than among the
Box 2: Definition of terms used in the current analysis

- Sensitivity - the percentage of pregnant women identified by the pregnancy exclusion criteria as possibly pregnant (pregnancy not ruled out).

- Specificity - the percentage of non-pregnant women for whom pregnancy is ruled out.

- Predictive value positive - the percentage of women identified by the checklist as possibly pregnant who are pregnant.

- Predictive value negative - the percentage of women identified by the checklist as non-pregnant who are not pregnant.

1830 clients $(1.3 \%)$ who tested negative. However, most women with signs or symptoms were not pregnant and would have been unnecessarily denied a contraceptive method using the current criteria.

Some signs and symptoms appeared to be more specific to pregnancy than others. For example, both clients for whom uterine masses were detected were pregnant, as was the one client who reported a combination of nausea and abdominal pain. Conversely, some classic symptoms of pregnancy, such as engorged breasts and missed periods, were not associated with any pregnancies. Only one symptom fell somewhere in between: one of 18 clients reporting nausea and/or vomiting was pregnant.

In two instances the signs and symptoms criterion 'detected' pregnancies that otherwise would have been ruled out by one of the six other criteria. Both were women with palpable uterine masses who reported having abstained from sexual relations for at least 6 months.

Figure 1 shows a post hoc comparison of outcomes when the pregnancy exclusion criteria are used with and without the signs and symptoms criterion, when each is compared with a commercial pregnancy test. Inclusion of the signs and symptoms criterion increases the number of pregnancies in the 'not ruled out' group from 12 to 14 (with a corresponding decrease in the number of 'false-negatives'). The trade-off for correctly excluding these two extra pregnant women is that pregnancy is not ruled out for about $1 \%$ more nonpregnant women; namely, in this sample, 18 additional non-pregnant women would inappropriately have had to wait for their next menstrual period or pay for a pregnancy test. In terms of test statistics, use of the signs and symptoms criterion increases the sensitivity of the criteria from 0.55 to 0.64 without any notable corresponding change in specificity or predictive value (Box 2). However, the confidence intervals of all the estimated test statistics, including sensitivity, overlap widely.

\section{Discussion}

In this population, the 'master criterion' did not substantially improve the ability of the algorithm to exclude pregnant clients. Moreover, for every extra

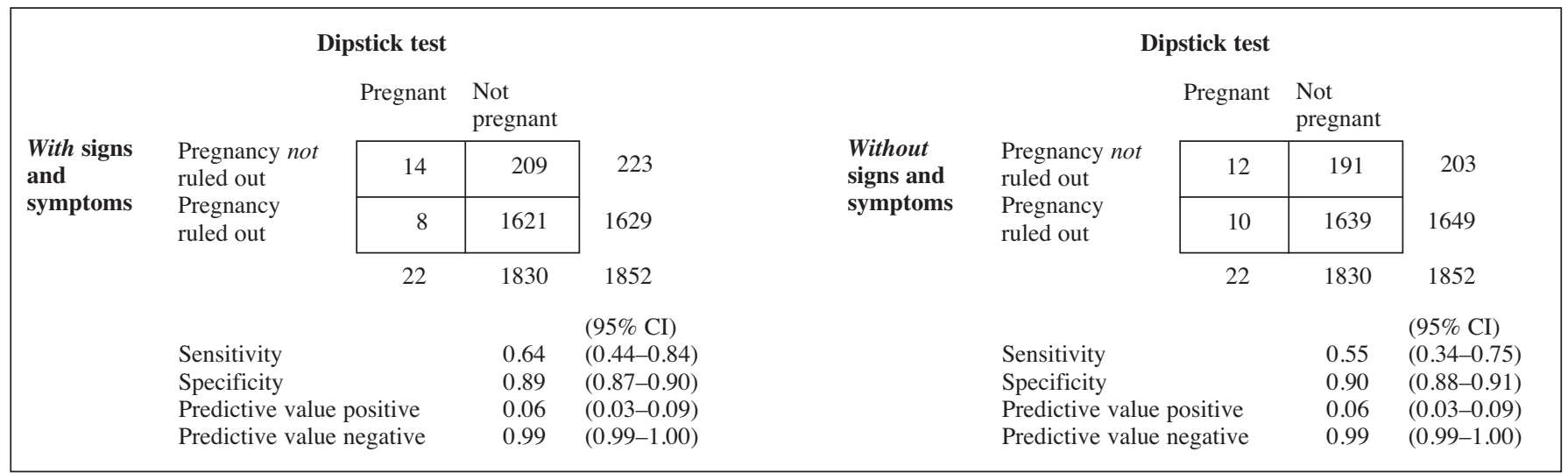

Figure 1 Comparison of pregnancy exclusion criteria with and without 'signs and symptoms' criterion 
pregnancy this criterion identified, nine non-pregnant women were effectively denied contraception.

Until more data are available, however, the signs and symptoms criterion will likely remain part of the algorithm for pregnancy exclusion. In the first place, the trade-off of nine clients sent home for each extra pregnancy detected would be considered justified in programmes where IUDs are common. And even where IUD use is rare, it is likely that exaggerated fears of the effects of fetal exposure to hormonal contraceptive methods could tip local opinion against any version of the algorithm without the signs and symptoms criterion, rendering it unacceptable to providers.

Could a compromise be found between including the current 'master criterion' and totally excluding it? Beyond the need to emphasise to providers the non-specificity of many traditional signs and symptoms, we think not. In the absence of new data from other regions and populations, we should focus our efforts on disseminating, rather than changing, this simple, effective algorithm. Women throughout the world continue to be denied family planning because of exaggerated provider fears about the prevalence and risks of undetected pregnancy. The real danger is needlessly exposing women to pregnancy, particularly in the poorest countries where women face a 1 in 15 lifetime risk of death associated with pregnancy and childbirth.
Acknowledgements

The authors are grateful for the helpful comments received from Barbara Janowitz, David Grimes, Jim Shelton and Rebecca Gmach.

Statements on funding and competing interests

Funding. Funding for this research was provided by the United States Agency for International Development (USAID) but the paper does not necessarily reflect USAID opinion.

Competing interests. None identified.

References

1 Stanback J, Thompson A, Hardee K, Janowitz B. Menstruation requirements: a significant barrier to contraceptive access in developing countries. Stud Fam Plann 1997; 28: 245-250.

2 World Health Organization (WHO). Selected Practice Recommendations for Contraceptive Use. Geneva, Switzerland: WHO, 2004.

3 Huezo CM, Carignan CS. Medical and Service Delivery Guidelines for Family Planning (2nd edn). London, UK: International Planned Parenthood Federation, 1997.

4 Hatcher R, Rinehart W, Blackburn R, Geller J, Shelton J. The Essentials of Contraceptive Technology. Baltimore, MD: Johns Hopkins University School of Public Health, Population Information Program, 1997

5 Stanback J, Qureshi Z, Sekadde-Kigondu C, Gonzalez B, Nutley T. Checklist for ruling out pregnancy among family-planning clients in primary care. Lancet 1999; 354: 566.

\title{
LETTERS TO THE EDITOR
}

Letters to the Editor are welcome and generally should not exceed 600 words or cite more than five references. For comments on material published in the most recent issue of the Journal, correspondence should be received within 4 weeks of dispatch of that Journal to be in time for inclusion in the next issue. When submitting letters correspondents should include their job title, a maximum of two qualifications and their address(es). A statement on competing interests should also be submitted for all letters. Letters may be submitted to the Editor or the Journal Editorial Office (details on page 1).

\section{termination of pregnancy}

\section{Respecting a woman's decision...}

\author{
A leading charity in the field of reproductive healthcare, Marie Stopes \\ International helps over one third of all women seeking termination of \\ pregnancy services in the UK each year. We work closely with the NHS and \\ also see private referrals.
}

We are discreet, confidential and supportive and offer expert care and full information. Women who visit us will have a full consultation and assessment and a choice of options including same day consultation and treatment, choice of surgical or medical abortion and choice of anaesthetics.

Patients can rely on our $\mathbf{2 4}$ hour aftercare service and post abortion counselling service.

\section{Call us on 08451203644 - 24 hours}

for a GP information pack or visit

www.mariestopes.org.uk 